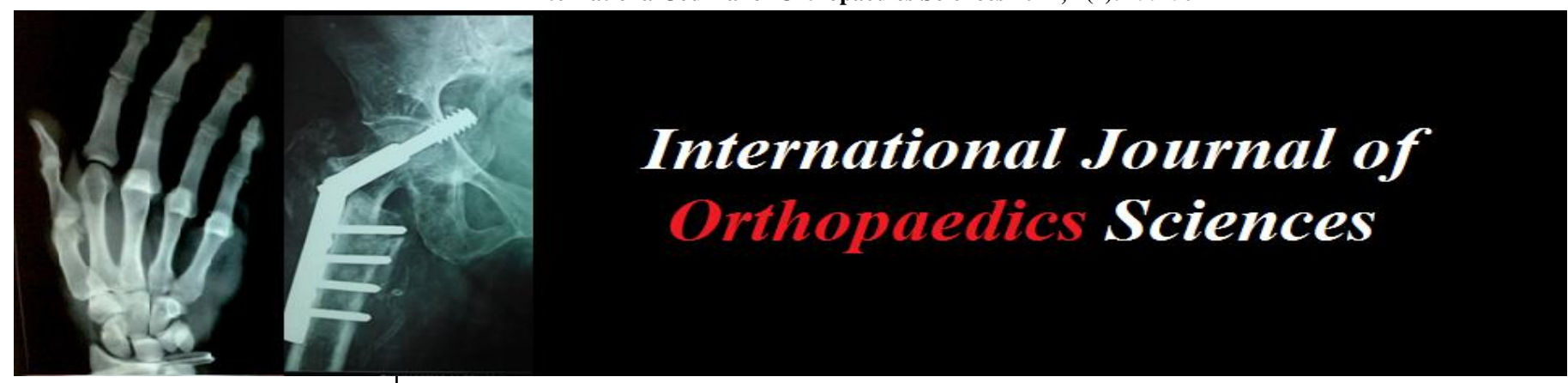

E-ISSN: 2395-1958

P-ISSN: 2706-6630

IJOS 2021; 7(2): 499-502

(C) 2021 IJOS

www.orthopaper.com

Received: 10-02-2021

Accepted: 17-03-2021

Dr. Akshay Bhimrao Fuse Resident, Department of Orthopaedics, Dr. Vasantrao Pawar Medical College, Nashik, Maharashtra, India

Dr. Shantanu Bhardwaj Resident, Department of Orthopaedics, Dr. Vasantrao Pawar Medical College, Nashik, Maharashtra, India

Dr. Aditya P Apte

Resident, Department of Orthopaedics, Dr. Vasantrao Pawar Medical College, Nashik, Maharashtra, India
Corresponding Author: Dr. Akshay Bhimrao Fuse Resident, Department of Orthopaedics, Dr. Vasantrao Pawar Medical College, Nashik, Maharashtra, India

\section{Treatment of in extra-articular distal tibia fracture Open vs Mippo which is better?}

\author{
Dr. Akshay Bhimrao Fuse, Dr. Shantanu Bhardwaj and Dr. Aditya P \\ Apte
}

DOI: https://doi.org/10.22271/ortho.2021.v7.i2g.2668

\section{Abstract}

Objective: Study was done to compare results between the Mippo and ORIF in extra articular distal tibia fracture. Hypothesis was made that outcome of MIPPO technique is comparable to ORIF.

Methodology: 30 patients were included in study out of which 15 patients were treated by Mippo technique and remaining 15 patients ORIF technique was used. Outcome was evaluated on basis of time of injury to surgery, operation time, blood loss, healing time, hospital stay, blood transfusion, implant related complication, radiation exposure, AOFAS grading.

Results: there was no remarkable difference in AOFS score, fracture union, hospital stay, mean operative time or wound healing. Blood loss was higher in ORIF group but radiation exposure was slightly more in MIPPO group, 1 case of malunion found in MIPPO group.

Conclusion: functional outcome of MIPPO technique are comparable with ORIF technique.

Keywords: Distal tibia fracture, ORIF, MIPPO, and Union

\section{Introduction}

Distal tibia fracture are one of the very common fracture witnessed in orthopaedics set up which occurs mainly in road traffic accidents and fall from height. Still the management of distal tibia fracture is in dispute amongst orthopaedics surgeon. Distal tibial anterio-medial surface subcutaneous location, minimal soft tissue coverage, deficient vascularity make it very demanding to treat the distal tibial fracture ${ }^{[1]}$. Historically distal tibia fractures were managed by closed reduction by means of cast and splints but rates of non-union, delayed union, malunion were high. In modern orthopaedics multiple surgical techniques have been described including intramedullary nailing, external fixation and plating ${ }^{[2]}$. Intramedullary nailing provides minimally invasive procedure, early fracture union and early mobilization but could proceed to malunion if nail is not centrally placed and snuggly fitted ${ }^{[3]}$. ORIF generally requires extensive soft tissue dissection and obvious periosteal stripping makes surroundings unfavorable for union ${ }^{[4]}$. In last decade new surgical technique has emerged 'MIPPO' which aims to minimize the soft tissue injury and periosteal stripping. Reduction is obtained percutaneosly without disturbing fracture hematoma in MIPPO, so it provides favorable environment for fracture healing by allowing micro motion at fracture site and maintaining vascularity of soft tissue ${ }^{[5]}$. With advancement of precontoured locking plates MIPPO gained popularity ${ }^{[6]}$. But still cases of malunion, delayed union, implant related problems are reported recently. Our objective to study the functional outcome of distal tibia fracture by two surgical procedure MIPPO VS ORIF assessed by clinical, social and radiological parameter.

\section{Material and Methodology}

Study was performed from July 2017 to Sept 2018, patients having distal tibia fracture were taken into account.

\section{Inclusion criteria}

1. Closed fracture of extra articular distal tibia with or without fibula fracture.

2. Skeletally matured patients.

3. No Neurovascular compromise. 
Informed written consent was taken from every patient

\section{Exclusion criteria}

1. Patients with open wound.

2. Pathological fracture.

3. Patients with incomplete follow-up.

4. Patients with polytrauma

Appropriate informed consent from patients and institutional ethics committee approval was taken. 30 patients with extra articular distal tibia fracture presenting to out patient's department/emergency department of our tertiary center were included in this study, they were divided into two groups of 15 patients each, and group 1 consisted of patients operated with ORIF and group 2 comprising of patients operated with MIPPO. The fracture configuration of each patients was further divided according to AO classification. And following data was collected:

All patients' detailed history noted, affected limb protected in above knee slab until the surgical intervention, anterior \& posterior x-ray taken. Limb elevation, magnesium sulfate dressings, ice application were given to lessen the soft tissue swelling. Patient was taken on operation table only when skin was pinchable overlying fracture site. All patients were treated with locking precontoured anatomical compression plates.

\section{Surgical technique}

All surgeries were performed in supine position on radiolucent table. High above knee tourniquet was used in all cases. 2 doses preoperative prophylactic third generation cephalosporin (cefuroxime $1.5 \mathrm{gm}$ iv) antibiotic were given to all cases on 12 hour before incision and other half hour before incision.

In ORIF conventional anteromedial incision taken starting from medial malleolus to proximal to it. Skin cut, subcutaneous cut, tried to prevent damage to periosteum, reduction was achieved by manipulation under vision. Precountured locking plate was placed over medial surface of distal tibia and reduction was secured with at least 4 proximal screws and 4 distal screws. In case of oblique and spiral fracture if needed one or two fully threaded cortical screw was inserted which acted as lag screw. Hemostasis was achieved after tourniquet deflation.

In MIPPO Technique: fracture was manipulated before taking an incision. Reduction was achieved closed under C -arm fluoroscopic guidance. Small straight incision taken longitudinally over center of medial malleolus precountured locking plate is slided proximally and subcutaneously without damaging the periosteum on medial surface. Reduction is temporarily fixed by 1 proximal and 1 distal $\mathrm{K}$ wire. Fixation is secured by 4 proximal and 4 distal screws to traction line. Proximal locking screws are placed by taking small $1 \mathrm{~cm}$ under fluoroscopic guidance.

Need for associated fibula fixation was assessed intraoperatively, on the basis of stability of inferior tibiofibular syndosmosis. If needed fibula was fixed by intramedullary nail.

\section{Postoperative management}

In no cases drain was placed under wound. Post-operative protocol was same for all cases. Knee ROM with quadriceps exercises was started on next day. Broad spectrum antibiotic coverage given for next 5 -7 days. Limb elevation with below knee slab was given. Patient was allowed to mobilize on walker once the swelling over distal is diminished. After radiological confirmation of callus formation gradual weight bearing on affected limb was started.

Follow up of all cases taken every 2 weeks and evaluated clinically and radio logically. Evaluation was assessed by AOFAS grading [7], union time, malunion, delayed union, non-union.

\section{Post-operative assessment}

Every case was followed for at least 6 months or more. Anterior posterior and lateral view x-ray of distal tibia was taken and follow up. Fracture union was defined radio logically when 3 or 4 cortices shows bridging callus in both $\mathrm{x}$ ray views and clinically when pain and tenderness at fracture site diminished.

When minimal sign of healing at fracture site seen even after 12 weeks post operatively, labeled as delayed union. Nonunion was defined as absence of any sign of healing within 24 weeks on plain $\mathrm{x}$-ray. Presence of any angular deformity more than $5^{\circ}$ and shortening more than $10 \mathrm{~mm}$, labeled as mal union. At end of follow up AOFAS score was used to assess the clinical outcome. Score of 90-100 was labeled as excellent, 75-89 as good, 50-74 as fair and $\leq 50$ as poor.

Two groups were compared on basis of time taken for admission to surgery, blood loss, blood transfusion, operative time, post op hospital stay, mean radiation exposure, any complications such as implant irritation or infection.

\section{Result}

No infection or implant loosening seen in either group. In MIPPO the mean age of patients was $48 \mathrm{yrs}$ (25-68yrs) and $40 y r s$ (22-55yrs). In MIPPO group 14 patients were male and single female patients and in ORIF all 15 patients were male only.

The mean interval from admission to surgery in ORIF group was 9 days (5-11 days) and while in MIPPO was also 9 days (7-15 days). Mean operative time was $113 \mathrm{~min}$ (90-150 min) in ORIF group and $110 \mathrm{~min}$ (90-140 min) in MIPPO group. This study being retrospective one it was difficult to measure the intraoperative blood loss but blood loss was calculated by soaked sponge count. Mean blood loss in MIPPO group was $139 \mathrm{ml}(100-300 \mathrm{ml})$ and $156 \mathrm{ml}(100-210)$ in ORIF group. One patient in each group required a single post-operative blood transfusion.

Mean post operatively hospital stay in ORIF group was 9 days (8-10days) and in MIPPO group was 10 days (8-12 days). Mean radiological union time in ORIF group was 14 weeks (10-20 weeks). and was 13.7 weeks (8-20weeks). No nonunion were encountered at follow up in either group.

Wound complication was observed in single patients in both the group. Which further required flap surgeries.

Mean AOFAS score in ORIF was 87.2 and in MIPPO group was 88.1

\section{Discussion}

Distal end tibia fractures are one of the most common cases encountered in the emergency department. The management options available includes non-operative, external fixation, intramedullary nail, MIPPO and conventional open plating ${ }^{(8)}$. Each of these modalities are associated with their own limitations. And the best line of management is controversial. Being subcutaneous in nature the distal tibia has minimal soft tissue coverage and precarious vascularity. Impaired blood supply due to damaged periosteum further delays fracture 
healing and increases the chances of nonunion ${ }^{[9]}$.

The principle of preservation of fracture hematoma and minimal soft tissue destruction is utilized for biological osteosynthesis in MIPPO technique ${ }^{[10]}$. This technique has shown successful results when clinical and radiological outcomes were compared. Conventional open plating is based on rigid internal fixation and stability achieved by maximum contact at fracture site ${ }^{[11]}$.

Complications associated with treatment of distal tibia such as delayed union and non-union are important comorbidities encountered. In studies conducted by $\mathrm{Li}$ et al. and Guo using MIPPO technique, they reported no incidence of delayed or non-union which was in accordance with results of our stud in the MIPPO group ${ }^{[12,13]}$.

As per recent reports, malunion has been described as a common complications $(2 \%-35 \%)$ in management of distal tibia fracture. In 88 distal tibia fracture treated with conventional open plating ${ }^{[14]}$, Lee et al. have noted only 3 $(3.4 \%)$ cases of malunion. In study by Zou et al, no malunion was reported using conventional open surgery [12, 15]. They have also reported that malunion have been seen in $9.6 \%$ of their patients treated with MIPPO technique. In a similar study conducted by Borg et al. the rate of malunion was reported to be $9.5 \%$. We encountered one case of malunion in MIPPO group ${ }^{[1]}$. Restoring perfect anatomical alignment in distal tibia fracture with MIPPO technique is challenging as reduction is achieved by indirect closed manipulation under fluoroscopic guidance that suggests reduction we get is acceptable.

Gulabi et al, shows no significant difference with respect to interval between time of injury and surgical intervention in either group, with concurrent reports by Wang Cheng et al. and similar outcomes in our study ${ }^{[12,16] .}$

ORIF is expected to have comparatively more blood loss because of extensive exposure. Wound complications and infection rate is expected to be higher. But we did not have any case of superficial or deep infection in case treated by ORIF. acurate anatomical reduction and rigid fixation is possible with ORIF. One case each of implant exposure due to wound dehiscence at medial malleolus was found in either group in our study. In our opinion, this is a limitation but which is not restricted to either of surgical technique, as the incision in both has to be taken over medial malleolus, which is the most subcutaneous part of the distal tibia and has very scanty blood supply and soft tissue coverage leading to wound dehiscence.

MIPPO group was found to have significantly lower blood loss compared to ORIF in our study, which is consistent with the findings reported by Cheng et al. Post-operative hospital stay in both groups have been found to be similar in study conducted by Cheng et al. ${ }^{[12]}$. Our study supports their findings.

The mean radiological union time in ORIF group was 14 weeks while that in MIPO was 13.7 weeks. Thus no significant difference was found in our study. These findings are comparable to that of Zou et al. and Gulabi et al. studies $[15,16]$.

After 6 months of follow up, in our study we found that the functional outcome score AOFAS, between the two groups was similar, which is supported by study conducted by Zou et al. and Gulabi et al. Ozsoy et al conducted studies on cadavers which have shown that MIPPO technique is associated with increased risk of injury to Saphenous nerve and Great saphenous vein ${ }^{[17]}$.

\section{Conclusion}

Thus in conclusion, both the techniques have their own merits and fallacies. While ORIF demands skillful surgical exposure with respect to raising soft tissue flaps to avoid necrosis and it leads to more intraoperative blood loss. MIPPO on the other hand needs less extensive soft tissue dissection but fracture reduction is more difficult to achieve. As reduction in MIPPO is dependent on fluoroscopy imaging, radiation exposure is also theoretically higher. So we conclude that the choice of surgery is dependent on surgeons training and experience.

ORIF: Open reduction and internal fixation.

MIPPO: Minimally invasive percutaneous plate osteosynthesis.

\section{Reference}

1. Borg T, Larsson S, Lindsjö U. Percutaneous plating of distal tibial fractures: preliminary results in 21 patients. Injury 2004;35(6):608-14.

2. Zelle BA, Bhandari M, Espiritu M, Koval KJ, Zlowodzki M. Evidence-Based Orthopaedic Trauma Working Group. Treatment of distal tibia fractures without articular involvement: a systematic review of 1125 fractures. Journal of orthopaedic trauma 2006;20(1):76-9.

3. Yang SW, Tzeng HM, Chou YJ, Teng HP, Liu HH, Wong CY. Treatment of distal tibial metaphyseal fractures: plating versus shortened intramedullary nailing. Injury 2006;37(6):531-5.

4. Im GI, Tae SK. Distal metaphyseal fractures of tibia: a prospective randomized trial of closed reduction and intramedullary nail versus open reduction and plate and screws fixation. Journal of Trauma and Acute Care Surgery 2005;59(5):1219-23.

5. Hasenboehler E, Rikli D, Babst R. Locking compression plate with minimally invasive plate osteosynthesis in diaphyseal and distal tibial fracture: a retrospective study of 32 patients. Injury 2007;38(3):365-70.

6. Gupta RK, Rohilla RK, Sangwan K, Singh V, Walia S. Locking plate fixation in distal metaphyseal tibial fractures: series of 79 patients. International orthopaedics 2010;34(8):1285-90.

7. Ibrahim T, Beiri A, Azzabi M, Best AJ, Taylor GJ, Menon DK. Reliability and validity of the subjective component of the American Orthopaedic Foot and Ankle Society clinical rating scales. The Journal of foot and ankle surgery 2007;46(2):65-74.

8. Bedi A, Le TT, Karunakar MA. Surgical treatment of nonarticular distal tibia fractures. JAAOS-Journal of the American Academy of Orthopaedic Surgeons 2006;14(7):406-16.

9. Collinge C, Protzman R. Outcomes of minimally invasive plate osteosynthesis for metaphyseal distal tibia fractures. Journal of orthopaedic trauma 2010;24(1):24-9.

10. Redfern DJ, Syed SU, Davies SJ. Fractures of the distal tibia: minimally invasive plate osteosynthesis. Injury. 2004;35(6):615-20.

11. Teeny SM, Wiss DA. Open reduction and internal fixation of tibial plafond fractures. Variables contributing to poor results and complications. Clinical Orthopaedics and related research 1993;(292):108-17.

12. Cheng W, Li Y, Manyi W. Comparison study of two surgical options for distal tibia fracture-minimally invasive plate osteosynthesis vs. open reduction and internal fixation. International Orthopaedics 2011;35(5):737-42. 
13. Guo JJ, Tang N, Yang HL, Tang TS. A prospective, randomised trial comparing closed intramedullary nailing with percutaneous plating in the treatment of distal metaphyseal fractures of the tibia. The Journal of bone and joint surgery. British 2010;92(7):984-8.

14. McCann PA, Jackson M, Mitchell ST, Atkins RM. Complications of definitive open reduction and internal fixation of pilon fractures of the distal tibia. International orthopaedics 2011;35(3):413-8.

15. Zou J, Zhang W, Zhang CQ. Comparison of minimally invasive percutaneous plate osteosynthesis with open reduction and internal fixation for treatment of extraarticular distal tibia fractures. Injury 2013;44(8):1102-6.

16. Gulabi D, Bekler Hİ, Saglam F, Tasdemir Z, Çeçen GS, Elmali N. Surgical treatment of distal tibia fractures: open versus MIPO. Ulus Travma Acil Cerrahi Derg 2016;22(1):52-7.

17. Ozsoy MH, Tuccar E, Demiryurek D, Bayramoglu A, Hayran M, Cavusoglu AT et al. Minimally invasive plating of the distal tibia: do we really sacrifice saphenous vein and nerve? A cadaver study. Journal of orthopaedic trauma 2009;23(2):132-8. 